\title{
Coprinopsis novorugosobispora (Basidiomycota, Agricales), ammonia fungus new to Canada
}

\section{Raut $\mathrm{JK}^{1}$, Fukiharu $\mathrm{T}^{2}$, Shimizu $\mathrm{K}^{3, *}$, Kawamoto $\mathrm{S}^{3}$, Takeshige $\mathrm{S}^{4}$, Tanaka $\mathrm{C}^{5}$, Yamanaka $T^{6}$ and Suzuki $A^{7}$}

\author{
${ }^{1}$ Nepal Academy of Science and Technology (NAST), Khumaltar, Lalitpur, GPO Box 3323, Kathmandu, Nepal \\ ${ }^{2}$ Natural History Museum and Institute, Chiba, 955-2, Aoba-cho, Chuo-ku, Chiba 260-8682, Japan \\ ${ }^{3}$ Medical Mycology Research Center, Chiba University, 1-8-1, Inohana, Chuo-ku, Chiba 260-8673, Japan \\ ${ }^{4}$ Faculty of Education, Chiba Universityl-33 Yayoi-cho, Inage-ku Chiba 263-8522, Japan \\ ${ }^{5}$ Graduate School of Agriculture, Kyoto University, Oiwake-cho, Kitashirakawa, Sakyo-ku, Kyoto 606-8502, Japan \\ ${ }^{6}$ Forestry and Forest Products Research Institute, Matsunosato, Tsukuba, Ibaraki 305-8687, Japan \\ ${ }^{7}$ Faculty of Knowledge Engineering, Tokyo City University1-28-1 Tamazutsumi, Setagaya-ku, Tokyo 158-8557 Japan \\ ${ }^{7}$ Faculty of Knowledge Engineering, Tokyo City University1-28-1 Tamazutsumi, Setagaya-ku, Tokyo 158-8557 Japan \\ *Present address: Department of Biological Science and Technology, Tokyo University of Science
}

Raut JK, Fukiharu T, Shimizu K, Kawamoto S, Takeshige S, Tanaka C, Yamanaka T, Suzuki A. 2015 - Coprinopsis novorugosobispora (Basidiomycota, Agricales), an ammonia fungus new to Canada. Mycosphere 6(5), 612-619, Doi 10.5943/mycosphere/6/5/10

\begin{abstract}
Fruiting of Coprinopsis novorugosobispora was effectively stimulated by urea treatment in the soil collected from aspen forest in Canada. A complete description and illustration on the fungus with its phylogenetic analysis based on nuclear rRNA gene sequences in ITS regions are provided. The report represents a new record in American continent.
\end{abstract}

Key words - Aspen - Coprinoid - saprobic - species complex - urea

\section{Introduction}

Ammonia fungi are defined as a chemoecological group of fungi that sequential occurrence is stimulated on natural soils by treatment of aqua ammonia or ammonia-releasing nitrogenous materials (Sagara 1975). To date, the study of ammonia fungi has been done in diverse geographical areas following application of urea in the field and/or laboratory such as in Australia (Suzuki et al. 1998, 2002a, 2003, Nagao et al. 2003, Fukiharu et al. 2011), Canada (Suzuki 2006, Raut et al. 2011), China (Fukiharu et al. 2012), Japan (Sagara and Hamada 1965, Sagara 1975, 1992, Suzuki 1992, Fukiharu and Hongo 1995, Yamanaka 1995a-c, Fukiharu \& Horigome 1996, Fukiharu et al. 1997, 2014, Sato \& Suzuki 1997, Suzuki et al. 1998, 2002b, 2003, Imamura 2001, He \& Suzuki 2004, Imamura \& Yumoto 2004, 2008, Sagara et al. 2008), New Zealand (Suzuki et al. 2002a, 2003, Fukiharu et al. 2011), Taiwan (Wang \& Sagara 1997), Thailand (Manusweeraporn et al. 2013), UK (Sagara et al. 2008), US (Sagara 1992) and Vietnam (Ho et al. 2014) ) and above 70 species of ammonia fungi have been recorded. However, ammonia fungi in boreal region have not yet been well investigated. We, therefore, surveyed ammonia fungi in boreal region near Edmonton, Canada by artificial urea application for the first time and collected a new taxon Coprinopsis neophlyctidospora Raut, Fukiharu \& A. Suzuki (Raut et al. 2011). This is the second report of the ammonia fungi in Basidiomycota from Canada. At the beginning it was reported as C. rugosobispora (J. Geesink \& Imler) Redhead, Vilgalys \& Moncalvo (Raut et al. 2010) but later based on the description of $C$. 
novorugosobispora Fukiharu \& Yamakoshi from China (Fukiharu et al. 2012) and detailed phylogenetic study with type specimens of former and latter both here it is described as $C$. novorugosobispora.

\section{Materials \& Methods}

\section{Isolates and morphology}

Basidiomata were obtained from the isolate incubated at $25^{\circ} \mathrm{C}$ on malt yeast agar medium (malt extract $10 \mathrm{~g} / \mathrm{l}$ (Difco, USA), yeast extract $2 \mathrm{~g} / \mathrm{l}$ (Difco, USA) and agar $15 \mathrm{~g} / \mathrm{l}$ (Nacalai Tesque, Japan) under lighting regime ( $12 \mathrm{~h}$ light/12 $\mathrm{h}$ dark). The isolate was obtained from a basidiomata collected on 8 July 2001 from the mixture of litter and soil collected from aspen forest (Populus tremuloides Michx.) followed by an application of urea (granular fertilizer; $46 \%$ nitrogen; $10 \mathrm{mg}$ $\mathrm{N} / \mathrm{g}$ dry soil) after 38 days of incubation at $25^{\circ} \mathrm{C}$ under lighting regime $(12 \mathrm{~h} \mathrm{light} / 12 \mathrm{~h}$ dark $)$ associating with watering about 2 days interval.

All descriptions of macro- and microscopic features were obtained from cultivated basidiomata. Anatomical observations and measurements were made on material mounted in $25 \%$ aqueous ammonia. Microscopic terminology of basidiospores and cystidia were followed on Vellinga (1988). Color terms and notations used in this description are based on Kornerup and Wanscher (1978). Herbarium abbreviations are according to Holmgren and Holmgren (1998). Basidiospore statistics: $x_{\mathrm{m}}$, the arithmetic mean of the spore length by spore width $( \pm$ standard deviation); n, number of spores measured; $\mathrm{Q}$, the quotient of spore length and spore width; $\mathrm{Q}_{\mathrm{m}}$, the mean of Q-values ( \pm standard deviation). For scanning electron microscopes (SEM) observation of basidiospore, samples were rehydrated in $25 \%$ aqueous ammonia and fixed in $2.5 \%$ osmium acid, coated with platinum-palladium sputter in an ion sputter-coater (Hitachi E-1030; Hitachi, Japan), and observed under a SEM (Hitachi S-800; Hitachi, Japan) operating at $15.0 \mathrm{kV}$.

\section{PCR Amplification and sequencing of ITS Region}

Fungal strains were grown in a MY (malt yeast extract) liquid medium. Mycelia were harvested, squeezed with a paper towel, frozen and lyophilized. The dried mycelia were then

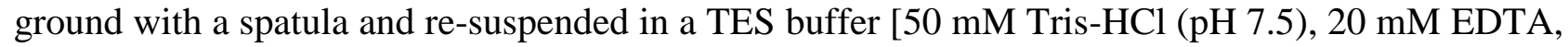
$1 \%$ SDS], and soluble fractions were recovered by the centrifugation. The DNA was purified by a TE buffer [10 mM Tris(HCl, pH 8.0)1 mM EDTA] saturated phenol/chloroform/isoamyl alcohol (Nippon gene, Japan) extraction followed by an iso-propyl alcohol precipitation. After desiccation of the DNA pellet, the DNA was dissolved in a $30 \mu \mathrm{l}$ TE buffer. For some samples, the genomic DNA was further purified by using a NucleoSpin Extract II (Macherey-Nagel, Germany) following the manufacture's recommendation. Primers ITS1 (5'-TCCGTAGGTGAACCTGTCGG-3') and ITS4 (5'-TCCTCCGCTTATTGTATGC-3') were used to amplify the ITS1-5.8S-ITS4 ribosomal DNA region (full ITS region). PCR reactions were carried out using the Ex Taq (TakaraBio, Japan) according to the manufacture's protocol. PCR products were purified by using the NucleoSpin Extract II, and the DNA fragments were directly sequenced using the BigDye Terminator v3.1 Cycle Sequencing Kit (Applied Biosystems, USA) according to the manufacturer's protocol. The reactions were then cleaned up using the Centri Sep (Princeton Separations) before analysis by the capillary electrophoresis on a 3130x DNA Analyzer (Applied Biosystems, USA). Sequences were assembled and edited using the ATSQ software (Genetyx, Japan). All nucleotide sequences were deposited in GenBank. The data set was aligned using Clustal X ver. 1.81 (Jeannmougin et al. 1998), and the resulting alignment was manually refined. The alignment was deposited in TreeBASE (http://www.treebase.org/) under the accession number http://purl.org/phylo/treebase/phylows/study/TB2:S16166. A phylogenetic tree was constructed based on the neighbor-joining (NJ) method (Saitou \& Nei 1987) using the NJ plot program (Perrière \& Gouy 1996). The robustness of inferred NJ topologies was tested by the bootstrap value (Felsenstein 1985) with 1000 replicates. Coprinopsis atramentaria (Bull.) Redhead, Vilgalys \& Moncalvo was used as the out group. Sequence data used from the GenBank are listed in the Table 1 with their accession numbers. 
Table 1 Taxa sampled for the phylogenetic analysis

\begin{tabular}{|c|c|c|c|c|}
\hline Taxa & Isolate No. & $\begin{array}{l}\text { Voucher specimen } \\
\text { No. }\end{array}$ & Locality & $\begin{array}{l}\text { GenBank } \\
\text { Accession No. }\end{array}$ \\
\hline \multicolumn{5}{|l|}{ Sequences generated in this study } \\
\hline \multirow[t]{2}{*}{ Coprinopsis novorugosobispora } & $\mathrm{SL}^{\mathrm{a}} 503001$ & CBM $^{\mathrm{d}}-\mathrm{FB} 38004$ & Alberta, Canada & AB564411 \\
\hline & & CBM-FB21471 & Beijing, China & AB978534 \\
\hline C. rugosobispora & & $\mathrm{Br}^{\mathrm{e}}-44338-09$ & Belgium & AB983245 \\
\hline \multicolumn{5}{|l|}{ Sequences retrieved from GenBank } \\
\hline \multirow[t]{3}{*}{ C. phlyctidospora (Romagn.) Redhead, Vilgalys \& Moncalvo } & $\mathrm{NBRC}^{\mathrm{b}} 30478$ & & Kyoto, Japan & $\mathrm{AB} 071615$ \\
\hline & & CBM-FB24542 & Kochi, Japan & $\mathrm{AB} 071613$ \\
\hline & & $1026^{\mathrm{f}}(\mathrm{Uljé})$ & & AB071608 \\
\hline \multirow[t]{3}{*}{ C. neophlyctidospora } & SL503201 & CBM-FB33899 & Alberta, Canada & AB564407 \\
\hline & SL503202 & CBM-FB33901 & Alberta, Canada & AB564406 \\
\hline & SL503203 & CBM-FB33894 & Alberta, Canada & AB564408 \\
\hline \multirow[t]{4}{*}{ C. austrophlyctidospora Fukiharu } & SL503401 & CBM-FB29564 & North Island, NZ & AB071793 \\
\hline & SL503402 & CBM-FB30247 & North Island, NZ & AB071795 \\
\hline & SL503403 & CBM-FB24556 & W. Australia & AB071796 \\
\hline & & E5808 ${ }^{\mathrm{g}}(\mathrm{CSIRO})$ & W. Australia & AB071791 \\
\hline \multirow[t]{4}{*}{ C. asiaticiphlyctidospora Fukiharu \& Horigome } & & CBMb-FB-38668 & Amami, Japan & AB818904 \\
\hline & & CBM-FB-37217 & Amami, Japan & AB817730 \\
\hline & & CBM-FB-37218 & Amami, Japan & AB817731 \\
\hline & & CBM-FB-37220 & Amami, Japan & $\mathrm{AB} 817732$ \\
\hline \multirow[t]{3}{*}{ C. echinospora (Buller) Redhead, Vilgalys \& Moncalvo } & & CBM-FB21629 & Aomori, Japan & AB071798 \\
\hline & & CBM-FB21725 & Aomori, Japan & AB071800 \\
\hline & & $537^{\mathrm{f}}$ (Uljé) & & AB071802 \\
\hline C. atramentaria & $\mathrm{KACC}^{\mathrm{c}} 49358$ & & & AF345814 \\
\hline
\end{tabular}

${ }^{a}$ SL numbers are stock cultures of Forestry and Forest Products Research Institute, Japan

${ }^{\mathrm{b}}$ NBRC: Culture collection of NITE Biological Resource Center, Japan

${ }^{\mathrm{c}}$ KACC: Culture collection of Korean Agricultural Culture Collection, Republic of Korea

${ }^{\mathrm{d}}$ CBM: Specimen collection of Natural History Museum and Institute, Chiba, Japan

${ }^{\mathrm{e}} \mathrm{Br}$ : Voucher Specimen stored in National Botanic Garden of Belgium

${ }^{\mathrm{f}}$ Voucher specimen stored by Dr. C.B. Uljé

${ }^{\mathrm{g}}$ Voucher specimen deposited in the Herbarium of CSIRO, Perth, Australia 


\section{Results}

\section{Coprinopsis novorugosobispora Fukiharu \& Yamakoshi in Mycoscience 54 (3): 226-230.}

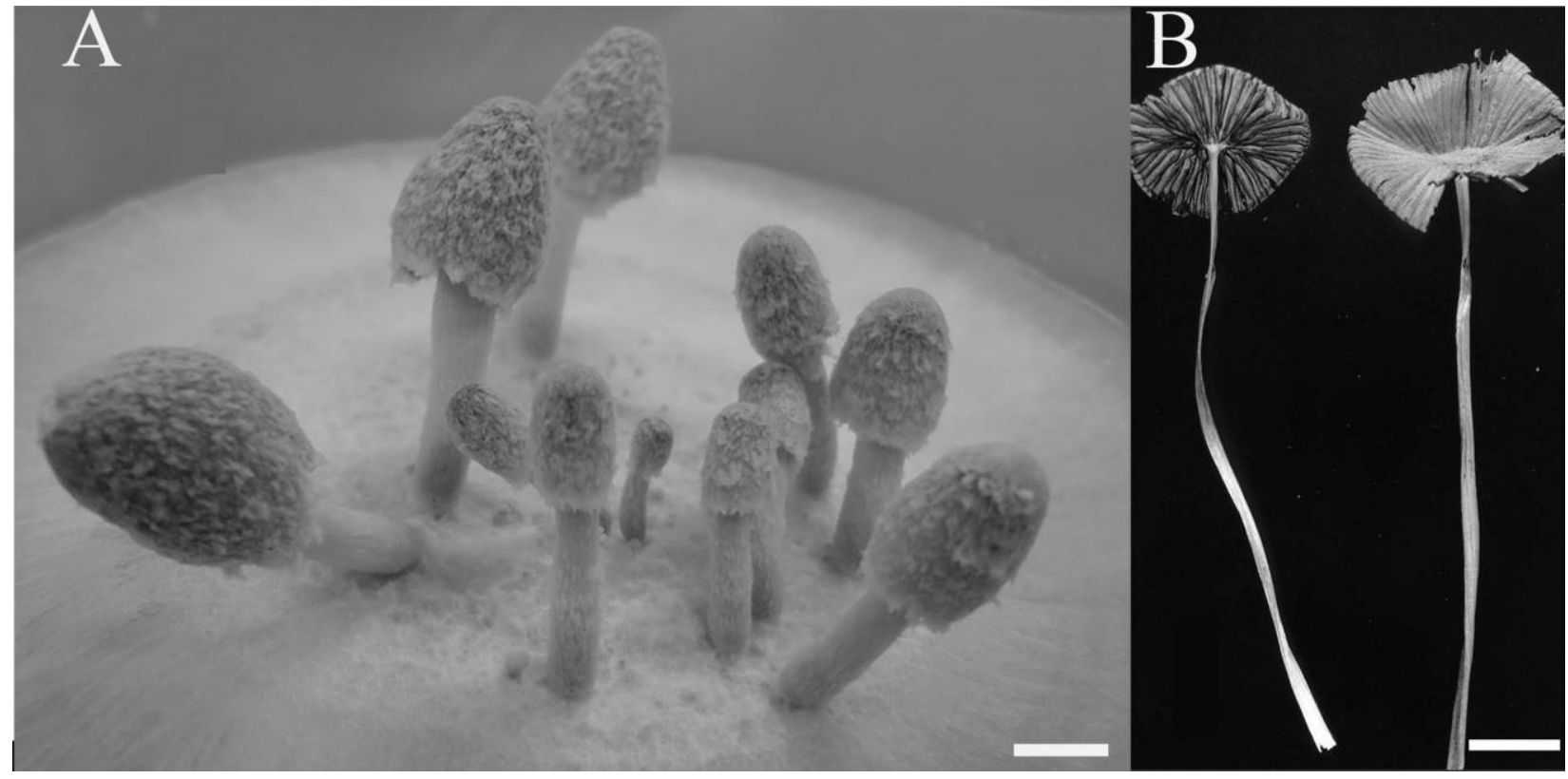

Fig. 1 - Macromorphology of Coprinopsis novorugosobispora collected from Canada. A-B Different developmental stages of basidiomata. Bars A-B $=5 \mathrm{~mm}$.

Description based on Canadian specimens

Pileus 4-15 $\mathrm{mm}$ broad, 3-10 $\mathrm{mm}$ when still closed, up to $25 \mathrm{~mm}$ when expanded, ellipsoid or ovoid when young, later convex to plane, finally uplifted. Pileipellis color at first white soon becoming gray, surface when young densely covered with white radially arranged hairy-fibrillose scales, later almost glabrous or veil remaining only in the center, flesh very thin, fragile. Lamellae free, close to crowded (number of lamellae 25-50), with 0-3 lamellulae between two lamellae, deliquescent, first white, then gray, finally blackish. Stipe $40-90 \times 1-3 \mathrm{~mm}$, central, cylindrical, not rooting, fistulose, fragile, base slightly clavate, surface white at first with fibrillose scales soon becoming smooth (Fig. 1A-B). Basidiospores black in mass, dark red-brown under microscope, 9.8-11.7 × 8.3-9.6 $\mu \mathrm{m}(\mathrm{xm}=10.7 \pm 0.4 \times 9.0 \pm 0.4 \mu \mathrm{m}, \mathrm{Q}=1.1-1.3, \mathrm{Qm}=1.2 \pm 0.1, \mathrm{n}=40)$, ovoid to ellipsoid with warty ornamentation, a central germ pore 1.6-2.3 $(2.0 \pm 0.3, \mathrm{n}=10) \mu \mathrm{m}$ wide, a clear plage (Fig. 3). Basidia 20-30 $\times 7-8 \mu \mathrm{m}$, one to two spored (Fig. 2A). Pleurocystidia 55-65 × 25-35 $\mu \mathrm{m}$, ellipsoid, utriform or broadly cylindrical, thin walled, hyaline (Fig. 2B-C). Cheilocystidia 35-55 × 20-30 $\mu \mathrm{m}$, sub-globose, ellipsoid, narrowly ovoid, utriform or broadly cylindrical (Fig. 2D). Elements of veil on the pileal surface composed of thin-walled, diverticulate, hyaline hyphae, 40-90 × 5-7 $\mu \mathrm{m}$ (Fig. 2E). Clamp-connections observed on vegetative hyphae.

Habit \& Habitat - Saprobic, solitary to gregarious, growing after application of urea $(10 \mathrm{mg}-$ N/g dry soil) in the soil collected from aspen (Populus tremuloides) forest in Canada.

Known Distribution - China, Canada

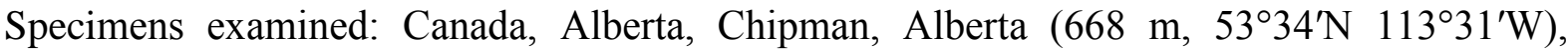
collected from the isolate incubated at $25^{\circ} \mathrm{C}$ on MY agar medium under lighting regime $(12 \mathrm{~h}$ light/12h dark). The isolate was obtained from a basidiomata occurred on urea amended forest soil of aspen ( $P$. tremuloides) A. Suzuki (CBM-FB 38004, CBM-FB 38005).

Other specimens examined: Coprinopsis rugosobispora: BR-44338-09 (Holotype, National Botanic Garden of Belgium, Geesink and Imler 1979). 


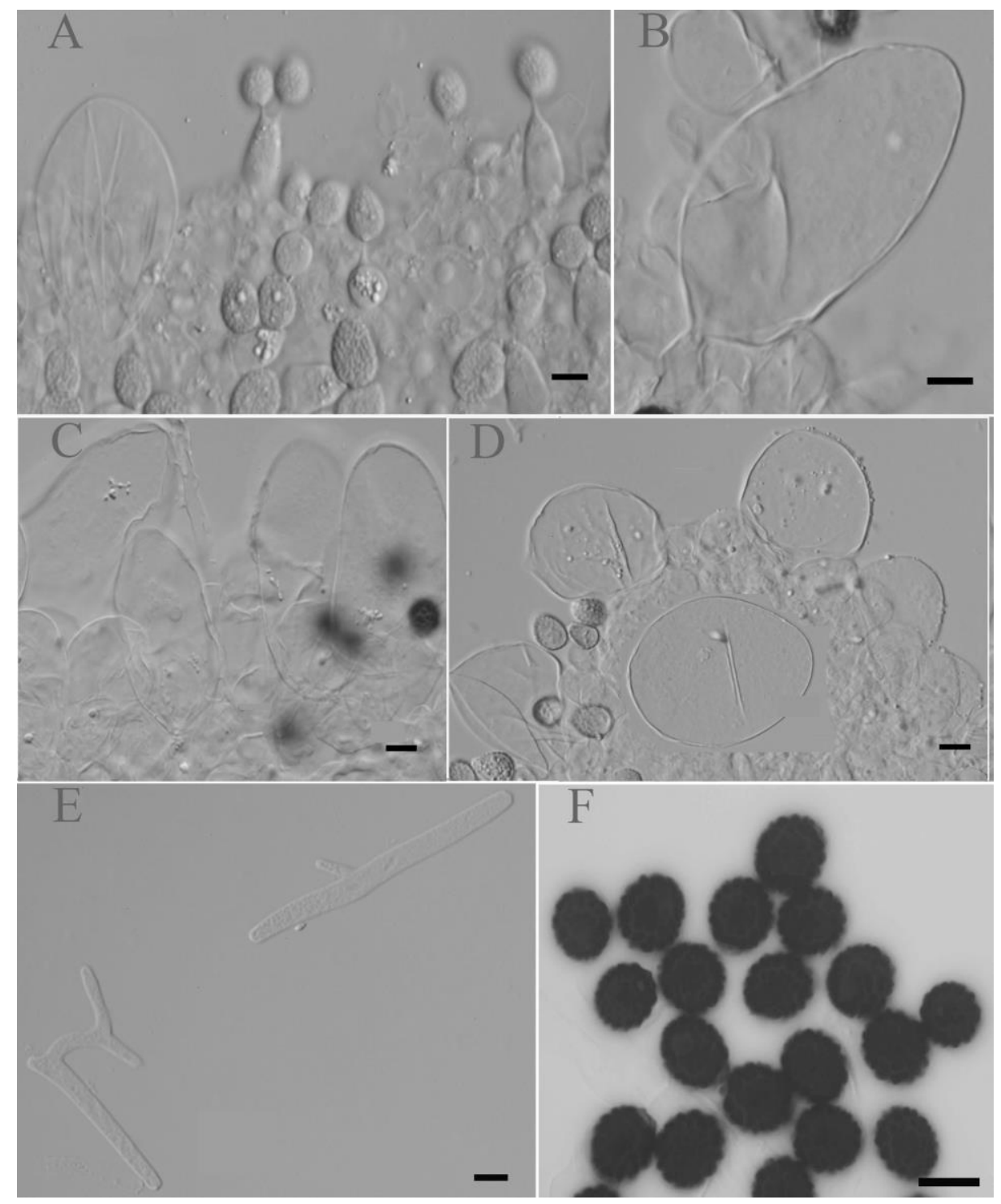

Fig. 2 - Micromorphology of Coprinopsis novorugosobispora collected from Canada. A: Basidia with basidiospores B-C: Pleurocystidia D: Cheilocystidia E: Veil elements F: basidiospores (under light microscope) Bars: $\mathrm{A}-\mathrm{F}=20 \mu \mathrm{m}$.

\section{Discussion}

The macro- and microscopic features of the Canadian specimen described above (Figs. 13 ), agree well with the original description of $C$. novorugosobispora from China (Fukiharu et al. 2012). Phylogenetic analysis also indicated that the samples from China and from Canada are placed in the same clade (Fig. 4). Coprinopsis novorugosobispora was phylogenetically clearly apart from $C$. rugosobispora with the high bootstrap value, but both species located in the group of C. phlyctidospora complex (Raut et al. 2011, Fukiharu et al. 2014). However, C. rugosobispora and $C$. phlyctidospora were not very well separated from each other (Fig. 4). Thus, more specimens should be determined to discuss whether C. rugosobispora (Geesink and Imler 1979) is an independent species or just a morphological variety of $C$. phlyctidospora. There have been a few records for the ammonia fungi (Amblyosporium botrytis Fresen., Ascobolus denudatus Fr., and Coprinopsis neophlyctidospora) in boreal forest of the North America (Suzuki 2006, Raut et al. 2011), and this report is the second one of Coprinopsis species from the boreal forest. In the preliminary study of ammonia fungi in the same forest, we have detected more saprobic species such as Coprinoid and Panaeolus spp., some ectomycorrhizal species such as Hebeloma spp. More study is necessary to reveal the complete community structure of the ammonia fungi in boreal forest in North America. 


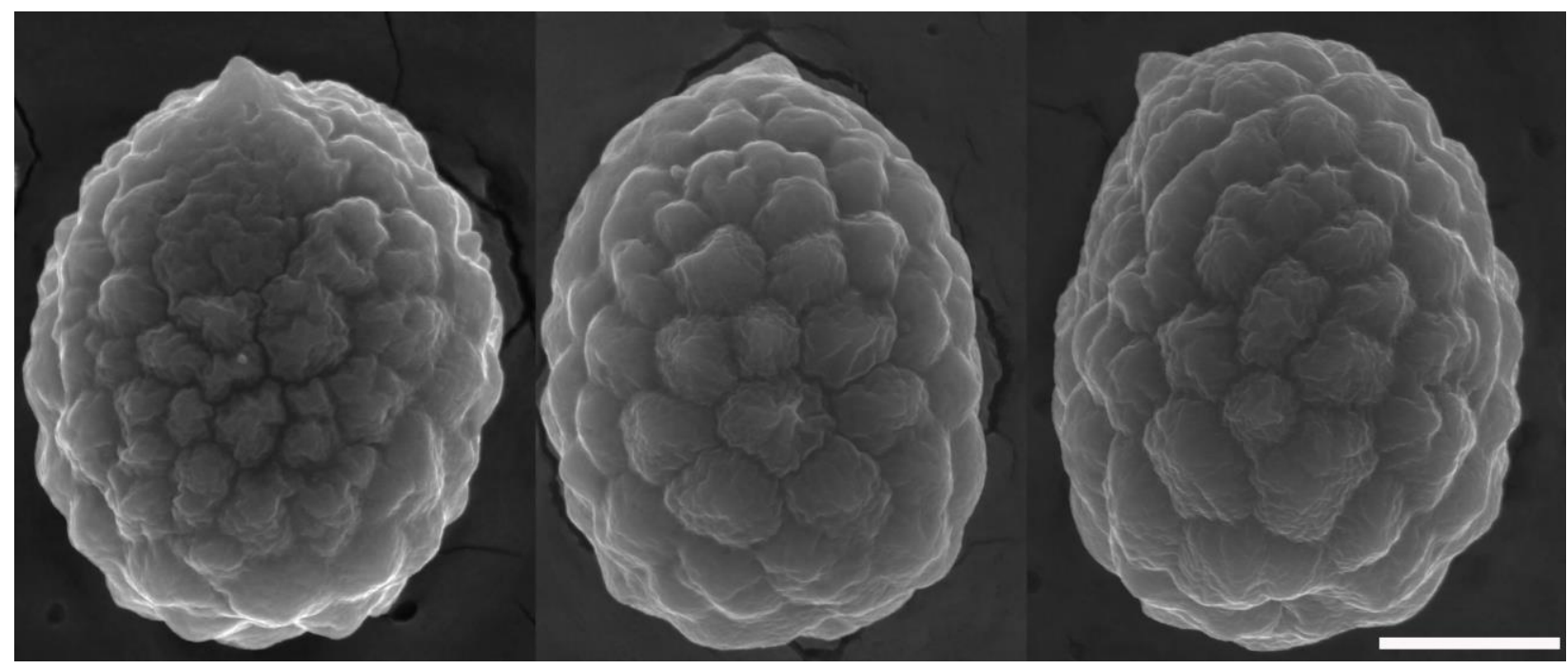

Fig. 3 - Basidiospores of Coprinopsis novorugosobispora collected from Canada. Face view, side view, back view from the left to right. Bar $=3 \mu \mathrm{m}$.

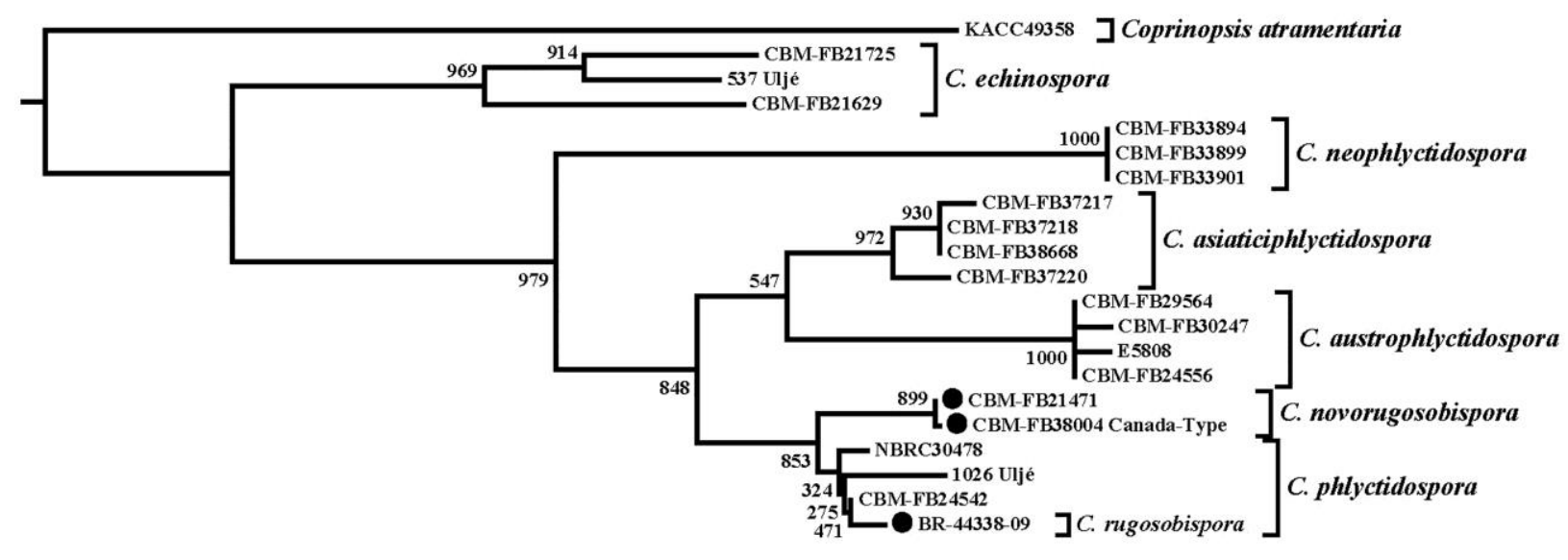

0.005

Fig. 4 - Phylogenetic tree constructed by the neighbor-joining (NJ) method (Saitou and Nei 1987) based on sequences of nuclear rRNA gene in ITS region. Numbers on the branches represent bootstrap values obtained from 1000 replication. The distance corresponding to five base changes per 1000 nucleotide position is indicated by the bar. Coprinopsis atramentaria was used as the outgroup taxon. Sidebar represents the inferred clades of Coprinopsis species. - Sequences generated in this study.

\section{Acknowledgements}

We are grateful to Prof. Jerôme Degreef, Head Curator of Cryptogams of National Botanic Garden of Belgium for use of type specimen. This work was financially supported in part by a Grant-in-Aid for the Scientific Research (Nos. 14560112 and 16570073) from the Japan Society for the Promotion of Science (JSPS). We would like to express sincere thanks to Dr. Randy S. Currah (Professor Emeritus of University of Alberta, Canada) for his making the experimental sites available and support field and laboratory experiments in Canada. Prof. Dev Narayan Yadav, an English language expert (Department of English, Patan Multiple Campus, Tribhuvan University, Nepal) is also greatly acknowledged for his valuable input in the manuscript. 


\section{References}

Felsenstein J. 1985 - Confidence limits on phylogenies: an approach using bootstrap. Evolution 39: 783-791.

Fukiharu T, Bougher NL, Buchanan PK, Suzuki A, Tanaka C, Sagara N. 2011 - Coprinopsis austrophlyctidospora sp. nov., an ammonia fungus from Southern Hemisphere plantations and natural forests. Mycoscience 52: 137-142; http://dx.doi.org/ 10.1007/s10267-010-00770.

Fukiharu T, Hongo T. 1995 - Ammonia fungi of Iriomote Island in the southern Ryukyus, Japan and a new ammonia fungus, Hebeloma luchuense. Mycoscience 36: 425-430.

Fukiharu T, Horigome R. 1996 - Ammonia fungi in the Abukuma Mountains and its biogeographical distribution around Japan (in Japanese with English summary). Memoirs of the National Science Museum 29: 105-112.

Fukiharu T, Sato Y, Suzuki A. 1997 - The occurrence of ammonia fungi, and changes in soil conditions and decay rate of bamboo in response to application of a large amount of urea in a bamboo grove in Chiba Prefecture, central Japan. The Bulletin of the Faculty of Education, Chiba University, 45 (III: Natural Sciences): 61-67.

Fukiharu T, Shimizu K, Li R, Raut JK, Yamkoshi S, Horie Y, Kinjo N. 2012 - Coprinopsis novorugosobispora sp. nov., an agraric ammonia fungus from Beijing, China. Mycoscience 54: 226-230; http://dx.doi.org/10.1016/j.myc.2012.09.014.

Fukiharu T, Shimizu K, Utsunomiya H, Raut JK, Goto R, Okamoto T, Kato M, Horigome R, Furuki T, Kinjo N. 2014 - Coprinopsis asiaticiphlyctidospora sp. nov., an agaric ammonia fungus from Amami and Okinawa, southern Japan. Mycoscience 55: 355-360; http://dx.doi.org/10.1016/j.myc.2013.12.002.

Geesink DJ, Imler L. 1979 - Coprinus rugosobisporus nov. sp. Sterbeeckia 12: 7-9.

He XM, Suzuki A. 2004 - Effects of urea treatment on litter docomposition in Pasania edulis forest soil. Journal of Wood Science 50: 266-270; http://dx.doi.org/ 10.1007/s10086-003-0546-6.

Ho BTQ, Pham N-DH, Shimizu K, Fukiharu T, Truong BN, Suzuki A. 2014 - The first record of Hebeloma vinosophyllum (Strophariaceae) in Southeast Asia. Mycotaxon 128: 25-36; http://dx.doi.org/10.5248/128.25.

Holmgren PK, Holmgren NH. 1998 - Index Herbariorum: A global directory of public herbaria and associated staff. New York Botanical Garden's Virtual Herbarium. http://sweetgum.nybg.org/ih/.

Imamura A. 2001 - Report on Laccaria amethystina, newly confirmed as an ammonia fungus. Mycoscience 42: 623-625.

Imamura A, Yumoto T. 2004 - The time of urea treatment and its effects on the succession of ammonia fungi in two warm temperature forests in Japan. Mycoscience 45: 123-130.

Imamura A, Yumoto T. 2008 - Dynamics of fruit-body production and mycorrhiza formation of ectomycorrhizal ammonia fungi in warm temperate forests in Japan. Mycoscience 49: 4255.

Jeannmougin F, Thompson JD, Gouy M, Higgins DG, Gibson TJ. 1998 - Multiple sequence alignment with Clustal X. Trends in Biochemical Sciences 23: 403-405.

Kornerup A, Wanscher JH. 1978 - Methuen Handbook of color, 3rd edn. Sankt Jorgen Tryk Ltd, Copenhagen.

Manusweeraporn S, Raut JK, Aramsirirujiwet Y, Kitpreechavanich V, Suzuki A. 2013 - The variation of litter decomposing abilities of Coprinopsis cinerea from nitrogen environments in Thailand. Thai Journal of Botany 5(Special Issue): 89-98.

Nagao H, Udagawa S, Bougher NL, Suzuki A, Tommerup IC. 2003 - The genus Thecotheus (Pezizales) in Australia: T. urinamans sp. nov. from urea-treated jarrah (Eucalyptus marginata) forest. Mycologia 95: 688-693.

Perrière G, Gouy M. 1996 - WWW-Query: an on-line retrieval system for biological sequences banks. Biochimie 78: 364-369. 
Raut JK, Suzuki A, Fukiharu T, Shimizu K, Kawamoto S, Tanaka C. 2011 - Coprinopsis neophlyctidospora sp. nov., a new ammonia fungus from boreal forests in Canada. Mycotaxon 115: 227-238; http://dx.doi.org/10.5248/115.227.

Raut JK, Suzuki A, Fukiharu T, Shimizu K, Tanaka C, Takeshige S. 2010 - A new record of an ammonia fungus, Coprinopsis rugosobispora collected from urea-treated boreal forest in Canada. The 6th meeting of East Asia for Collaboration on Edible Fungi, Gwangju, Korea.

Sagara N. 1975 - Ammonia fungi: a chemoecological grouping of terrestrial fungi. Contributions from the Biological Laboratory, Kyoto University 24: 205-276.

Sagara N. 1992 - Experimental disturbances and epigeous fungi. In: Carroll GC, Wicklow DT (eds) The fungal community: its organization and role in the ecosystem, 2nd $\mathrm{ed}^{\mathrm{n}}$. Marcel Dekker, New York, pp 427-454.

Sagara N, Hamada M. 1965 - Responses of higher fungi to some chemical treatments of forest ground. Transactions of the Mycological Society of Japan 6: 72-74.

Sagara N, Yamanaka T, Tibbett M. 2008 - Soil fungi associated with graves and latrines: Toward a forensic mycology. In Tibbett M, Carter DO (eds) Soil analysis in forensic taphonology, CRC Press, Boca Raton, pp 67-107.

Saitou N, Nei M. 1987 - The neighbor-joining method: a new method for reconstructing phylogenetic trees. Molecular Biology and Evolution 4: 406-425.

Sato Y, Suzuki A. 1997 - The occurrence of ammonia fungi, and changes in soil conditions and wood decay rate in response to application of a large amount of urea in a Quercus serrata dominated mixed forest in Meguro, Tokyo. The Bulletin of the Faculty of Education, Chiba University, 45 (III: Natural Sciences): 53-59.

Suzuki A. 1992 - Physiological characteristics of the ammonia fungi and their role in bioscience. Proceedings of the Asian Mycological Symposium, Seoul, ROK. pp 238-247.

Suzuki A. 2006 - Experimental and physiological ecology of ammonia fungi: studies using natural substrates and artificial media. Mycoscience 47: 3-17; http://dx.doi.org/10.1007/s10267005-0270-8.

Suzuki A, Fukiharu T, Tanaka C, Ohono T, Buchanan PK. 2003 - Saprobic and ectomycorrhizal ammonia fungi in the Southern Hemisphere. New Zealand Journal of Botany 41: 391-406; http://dx.doi.org/10.1080/ 0028825X.2003.9512858.

Suzuki A, Tanaka C, Bougher NL, Tommerup IC, Buchanan PK, Fukiharu T, Tsuchida S, Tsuda M, Oda T, Fukada J, Sagara N. 2002a - ITS rDNA variation of the Coprinopsis phlyctidospora (Syn.: 229-238; Coprinus phlyctidosporus) complex in the Northern and Southern Hemispheres. Mycoscience 43: http://dx.doi.org/ 10.1007/s102670200033.

Suzuki A, Tommerup IC, Bougher NL. 1998 - Ammonia fungi in the Jarrah forest of Western Australia and parallelism with other geographic regions of the world. Proceedings of Biodiversity, Biotechnology \& Biobusiness 2nd Asia-Pacific Conference on Biotechnology (Sheraton Perth Hotel), 23-27 November, 1998. p127.

Suzuki A, Uchida M, Kita M. 2002b - Experimental analyses of successive occurrence of ammonia fungi in the field. Fungal Diversity 10: 141-165.

Vellinga EC. 1988 - Glossary. In: Bas C, Kuyper TW, Noordeloos ME, Vellinga EC, (eds.) Flora Agaricina Neerlandica. Vol. 1. A. Balkema, Rotterdam, pp 54-64.

Wang YZ, Sagara N. 1997 - Peziza urinophila, a new ammonophilic discomycete. Mycotaxon 65: 447-452.

Yamanaka T. 1995a - Changes in organic matter composition of forest soil treated with a large amount of urea to promote ammonia fungi and the abilities of these fungi to decompose organic matter. Mycoscience 36: 17-23.

Yamanaka T. 1995b - Nitrification in a Japanese red pine forest soil treated with a large amount of urea. Journal of the Japanese Forest Society 77: 232-238.

Yamanaka T. 1995c - Changes in soil conditions following treatment with a large amount of urea to enhaunce fungal fruiting-body production in a Japanese red pine forest. Bulletin of Japanese Society of Microbial Ecology 10: 67-72. 